\title{
Dynamic Contrast Enhanced (DCE) MRI-Derived Renal Perfusion and Filtration: Experimental Protocol
}

\author{
Pietro Irrera, Lorena Consolino, Walter Dastrù, Michael Pedersen, \\ Frank G. Zöllner, and Dario Livio Longo
}

\begin{abstract}
Dynamic contrast-enhanced magnetic resonance imaging (DCE-MRI) can provide a noninvasive way for assessing renal functional information following the administration of a small molecular weight gadolinium-based contrast agent. This method may be useful for investigating renal perfusion and glomerular filtration rates of rodents in vivo under various experimental (patho)physiological conditions. Here we describe a step-by-step protocol for DCE-MRI studies in small animals providing practical notes on acquisition parameters, sequences, $\mathrm{T}_{1}$ mapping approaches and procedures.

This chapters is based upon work from the COST Action PARENCHIMA, a community-driven network funded by the European Cooperation in Science and Technology (COST) program of the European Union, which aims to improve the reproducibility and standardization of renal MRI biomarkers. This experimental protocol chapter is complemented by two separate chapters describing the basic concept and data analysis.
\end{abstract}

Key words Magnetic resonance imaging (MRI), Kidney, Mice, Rats, Dynamic contrast-enhanced (DCE), Contrast agent, $\mathrm{T}_{1}$ map

\section{Introduction}

Kidneys play an important role in fluid regulation, waste products elimination, and homeostasis of salts and $\mathrm{pH}$. Therefore, accurate measurement of kidney function is essential for monitoring early renal injuries, disease progression, renal transplantation and in assessing therapeutic efficacy after renal failure [1, 2]. Magnetic resonance imaging (MRI) has emerged as a powerful technique that combines anatomical details with functional information. In recent years, various MRI techniques have been investigated for assessing renal function, including dynamic contrast-enhanced (DCE) MRI, arterial spin labelling (ASL), blood oxygenation level dependent (BOLD), diffusion weighted imaging (DWI), sodium imaging, chemical exchange saturation 
transfer (CEST), and hyperpolarized MRI [3-9]. These techniques have shown promising results for the noninvasive evaluation of several renal function, including filtration, perfusion, oxygenation, $\mathrm{pH}$, homeostasis, and others [10-27]. Among them, DCE-MRI or MR renography has emerged as a reliable method to reveal renal pathophysiology, functional and hemodynamic parameters. DCE-MRI relies on bolus administration of a gadolinium-based contrast agent (CA) [16], which changes the intrinsic longitudinal $\left(\mathrm{T}_{1}\right)$ and transversal $\left(\mathrm{T}_{2}\right)$ relaxation rates of protons in tissue. This means that administration of CA leads to an increased signal intensity on $\mathrm{T}_{1}$-weighted images or decreased signal intensity on $\mathrm{T}_{2}$-weighted images. DCE-MRI usually refers to $\mathrm{T}_{1}$-weighted MR imaging techniques combined with a gadolinium-based CA using an acquisition scheme with a high temporal resolution. In the kidney the acquired signal over time leads to an MRI renogram, where the absolute signal intensity, in complex manner, depends on the native $\mathrm{T}_{1}$ value, MRI sequence parameters, the specific magnetic relaxivity constant of the CA (the contrast agent efficiency, dependent also on its chemical structure [28-34]) and the concentration of the CA residing in the tissue $[35,36]$.

The main purpose of DCE-MRI is to reveal information about extravascular permeability, blood perfusion and compartmental kinetics. In practice, this method provides images prior and following the administration of the CA using a continuous image acquisition protocol. In kidneys, DCE-MRI is suitable for assessing kidney function in terms of filtration and perfusion [17]. Different postprocessing methods allow estimations of kidney function and perfusion, either from simple semiquantitative approaches to more elaborated model-based analyses [37-42].

Here we describe step-by-step an experimental dynamic contrast enhanced (DCE) MRI protocol for monitoring the passage of a Gd-based tracer in the kidneys of rodents. The rationale for the choosing acquisition parameters is given in generic terms, together with specific parameter examples.

This experimental protocol chapter is complemented by two separate chapters describing the basic concepts and data analysis, which are part of this book.

This chapter is part of the book Pohlmann A, Niendorf T (eds) (2020) Preclinical MRI of the Kidney-Methods and Protocols. Springer, New York.

\section{Materials}

2.1 Animals
This experimental protocol was tailored for immune-competent (typically $\mathrm{C} 57 \mathrm{BL} / 6$ and Balb/C) or immune-compromised (nude, NOD-SCID) mice with a body mass of 15-35 g. Advice for adaptation to rats is given as Notes where necessary. All animal 
studies have to be carried out in compliance with specific legislation covering the use of animals for scientific purposes. Therefore, all experiments must be authorized under national regulations.

\subsection{Lab Equipment and Chemicals}

1. Mouse tail illuminator/restrainer for catheterizing the tail vein.

2. Saline solution or heparin solution, $1-\mathrm{ml}$ syringe, catheter (e.g., PE 20 polyethylene tubing) of known inner diameter to hold the saline flush and for contrast agent bolus is required.

3. Anesthesia: please refer to the chapter by Kaucsar $\mathrm{T}$ et al. "Preparation and Monitoring of Small Animals in Renal MRI" for an in-depth description and discussion of the anesthesia. Typically, $0.5-1.5 \%$ isoflurane is used for anesthesia administered to the mice using an anesthetic gas vaporizer (Leica Biosystems, Maryland, USA). For nonrecovery experiments, urethane solution (Sigma-Aldrich, Steinheim, Germany; $20 \%$ in distilled water) can provide anesthesia for several hours with comparatively fewer side effects on renal physiology, which is an important issue. For intramuscular anesthesia, please refer to Note $\mathbf{1}$.

4. Gases: $\mathrm{O}_{2}, \mathrm{~N}_{2}$, and compressed air, as well as a gas-mixing system or general inhalation anesthesia equipment, including an anesthetic vaporizer, a flow meter, and an induction chamber.

5. Mouse cradle.

6. Gd-based contrast agents: typically, small molecular weight FDA-approved gadolinium-based contrast agents are employed, such as gadoteridol (Prohance ${ }^{\circledR}$, Bracco Imaging SpA, Milano, Italy), gadobutrol (Gadovist ${ }^{\mathbb{B}}$, Bayer Healthcare Pharmaceuticals, Germany), or gadoteric acid (Dotarem ${ }^{\circledR}$, Guerbert, France). Such clinically available contrast agents have stock concentrations of $0.5-1.0 \mathrm{~mol} / \mathrm{l}$ that need to be diluted for handling volumes with less error (see Note 2).

7. Small animal ventilator: animals (rats) needs to be ventilated so the DCE-MRI acquisition can be gated to minimize the respiratory motion artifacts.

8. Syringe infusion pump: an MRI-compatible single syringe pump (Harvard Bioscience, Holliston, USA) for contrast agent administration for allowing precise timing infusion.

\subsection{MRI Hardware}

The general hardware requirements for renal $1 \mathrm{H}$ MRI on mice and rats are described in the chapter by Ramos Delgado P et al. "Hardware Considerations for Preclinical Magnetic Resonance of the Kidney." The technique described in this chapter was tailored for MRI preclinical systems at a magnetic field strength of $7 \mathrm{~T}$ but advice for adaptation to other magnetic field strengths is given where necessary ( see Note 3). No special or additional hardware is required, except for the following: 


\subsection{MRI Techniques}

1. A physiological monitoring system that can track the respiration and which is connected to the MRI system such that it can be used to synchronize DCE-MRI with respiration. Typically, we use an MRI-compatible rodent monitoring and gating system equipped with an air-pillow to monitor breath rate (Small Animal Instruments, New York, USA).

DCE-MRI experiments are performed by dynamic acquisitions of a strongly $\mathrm{T}_{1}$-weighted imaging technique. The acquired signal following the CA administration is converted to relative concentrations of the gadolinium agent using either phantom calibration scales or by mathematical calculation of the sequence equation that describes the relationship between signal and MRI sequence parameters. The MRI sequence parameters, required volume coverage, spatial resolution, and SNR will determine the final temporal resolution. Several $T_{1}$ mapping approaches can be exploited for measuring the longitudinal relation times, a detailed description of different methods is provided in the chapter by Garteiser P et al. "Experimental Protocols for MRI Mapping of Renal $\mathrm{T}_{1}$."

\section{Methods}

\subsection{MR \\ Protocol Setup}

3.1.1 Variable Repetition

Time (VTR) Acquisition

for $T_{1}$ Mapping
1. Use a 2D RARE (rapid acquisition with refocused echoes) sequence with variable repetition times (RAREVTR on a Bruker MR system).

2. Set the acceleration factor (RARE-factor or echo train length, ETL) to at least 4 in order to shorten the acquisition time; an acceleration factor of 8-16 can be used to further shorten the acquisition time by sampling more $\mathrm{k}$-space lines within a single repetition period (TR). Centric encoding can be exploited to minimize $\mathrm{T}_{2}$ effects with increasing doses, but at the expenses or "blurred" images.

3. Set the echo time (TE) as short as possible for pure $\mathrm{T}_{1}$-weight; in our setup an effective TE of $25 \mathrm{~ms}$ (defined by the acquisition of the central k-space line) was used.

4. Choose enough averages in order to have a sufficient signal to noise ratio (SNR) of above 30 .

5. Repeat the RARE acquisition a number of times by changing the value of the repetition time (TR) to sample changes in signal intensities according to the expected $T_{1}$ values of the kidneys.

6. Because of the variability of the $T_{1}$ values inside the kidney regions, choose the TRs values to span a range large enough to permit an accurate calculation of $\mathrm{T}_{1}$ by selecting $3-7 \mathrm{TR}$ values in the range 100-10,000 ms (see Note 4). 
3.1.2 Variable Flip Angle (VFA) Acquisition for $T_{1}$ Mapping

3.1.3 Fast Low Angle Shot (FLASH) T1-Weighted Sequence for DCE-MRI Experiments
7. For in vivo experiments, keep the overall acquisition time as short as possible by considering a trade-off between number of TRs, averages and matrix size (or spatial resolution).

8. For an example of a specific parameter set please see Note $\mathbf{5}$.

1. Use a 2D gradient-echo sequence (FLASH on a Bruker system).

2. Set the echo time (TE) as short as possible for heavily $T_{1}$ weighting; in our setup a TE of $1.5 \mathrm{~ms}$ was used.

3. In order to have a sufficient signal to noise ratio, at least two averages have been acquired or enough averages for $\mathrm{SNR}>30$.

4. Repeat the FLASH acquisition a number of times by changing the value of the flip angle (FA). The changes in signal intensities will follow a parabolic curve, thus low flip angles and higher flip angles are needed to sample both side of this curve. Acquire a minimum of 2-3 flip angles, considering that experiments with more flip angles will improve the goodness of the $T_{1}$ estimate.

5. Set the TR in the range $10-100 \mathrm{~ms}$ for providing good $\mathrm{T}_{1}$ weighting. Shorter TR increases $T_{1}$ weighting, but with detrimental SNR values; thus, a trade-off must be chosen, even considering number of averages and overall acquisition time.

6. For an example of a specific parameter set please see Note 6.

1. Use a $\mathrm{T}_{1}$-weighted sequence as a fast low angle shot (FLASH) sequence (in Bruker systems, this sequence refers to a dubbed FLASH sequence).

2. Use short $T R$ to increase $T_{1}$-weighting, but this will decrease SNR. Therefore, a good balance should be found between strongly $\mathrm{T}_{1}$-weighted images and image quality with sufficient SNR. Besides, TE and FA affect $\mathrm{T}_{1}$-weighting and SNR. A good TR range could be between 5 and $100 \mathrm{~ms}$ (usually shorter values for 3D imaging and longer for $2 \mathrm{D}$ multislice acquisition).

3. Use the shortest echo time (TE) possible for pure $T_{1}$ weight.

4. Flip Angle (FA): high FA corresponds to a strong $\mathrm{T}_{1}$ weighting, but at expenses of reduced SNR, therefore a good balance may result in FAs in the range $10-60^{\circ}$.

5. Use Partial Fourier acceleration to reduce the size of the acquired matrix and improve the time efficiency of data acquisition.

6. Use a high acquisition bandwidth (BW) to shorten TE, while keeping an eye on the SNR, which decreases with the square root of the increasing BW. For the image with the shortest TE an SNR of at least 30 is recommended. 
3.2 Preparations of the Contrast Agent Injection and of the Catheter

\subsection{Preparations of the Mouse}

7. Employ Fat Saturation modules. It is important to avoid fat signal overlaying with the kidney due to chemical shift.

8. Respiration trigger: there is no need to use respiratory triggering (per slice).

9. Adapt the geometry (or slice position) so that animal fits into FOV in Left-Right direction (approximately $30-40 \mathrm{~mm}$ according to the inner coil diameter) and use frequency encoding in Head-Feet direction. Use a slice with the lowest thickness the SNR allows, typically around $1-2 \mathrm{~mm}$. The thinner the slices the better, as this improves the resolution which can be readily found on the raw images.

10. Use the highest in-plane resolution that the SNR allows, typically around $96 \times 96$ to $128 \times 128$ for FOV of $30 \times 30 \mathrm{~mm}^{2}$ or $40 \times 40 \mathrm{~mm}^{2}$. This resolution should be balanced by the need for sufficient SNR in the images. Zero-filling or use of partial Fourier techniques in the phase encoding direction can be helpful to speed up acquisition to minimize motion artifacts and contrast agent concentration changes during the acquisition. One may use half-Fourier acquisition schemes in read direction (asymmetric echo) to further shorten the TE. Reducing the excitation and refocusing pulse lengths to below $1.5 \mathrm{~ms}$ can then also help to shorten TE.

11. For an example of specific parameter sets for a 7 T MRI system, please see Notes 7 and $\mathbf{8}$, whereas for a scanner operating at $3 \mathrm{~T}$ please see Note 9.

1. Fill the 1-ml syringe with the contrast agent solution (see Note 2).

2. Prepare the catheter by filling the PE20 tubing with the contrast agent solution, and with 20-40 $\mu \mathrm{l}$ of a heparin solution (or a saline solution), between the needle (the animal) and the contrast agent solution to prevent clotting before the contrast agent will be injected.

3. According to the length of the catheter (usually $60-100 \mathrm{~cm}$ to exit from the magnet bore) the contrast agent solution can be injected directly from the syringe containing the contrast agent or by changing the syringe with one filled with a (heparin-free) saline solution for the postinjection flush.

1. Place the mouse on a mouse tail illuminator restrainer for catheterizing the tail vein. The tail vein can be catheterized by placing a $27 / 29-\mathrm{G}$ needle in the animal.

2. Induce anesthesia in animals via isoflurane in an induction box. Then transfer the animal to the MRI system ( see Note 10).

3 . Set up the temperature monitoring (rectal probe) and respiratory monitoring (balloon on chest) unit. During imaging, keep 
the animal's respiration rate at $35-80$ breaths per min by adjusting the dose of anesthesia (approximately $1-1.5 \%$ isoflurane with air and oxygen mixed at a 3:1 ratio administered through a nose cone attached to the animal bed and ophthalmic ointment will be applied to the eyes) ( see Note 11).

4. Perform anatomical imaging as described in the chapter by Pohlmann A et al. "Essential Practical Steps for MRI of the Kidney in Experimental Research."

5. Since the AIF should be sampled with the same temporal resolution of the DCE-MRI experiment, selection of anatomical slices including large vessels near the kidneys should be considered.

6. Perform localized shimming on the kidney imaging as described in the chapter by Pohlmann A et al. "Essential Practical Steps for MRI of the Kidney in Experimental Research."

\subsection{In Vivo $T_{1}$ Mapping}

3.4.1 DCE-MRI Experiment
Typically, a $T_{1}$ map is acquired before the injection of Gd-based agent.

1. Select the same geometry of the anatomical images, with the same number of slices and position.

2. Load or select one of the several $T_{1}$ mapping sequences previously described (3.1). Adapt the same slice orientation as for the anatomical image with coronal or axial view with respect to the kidneys.

3 . Run the $T_{1}$-mapping scan. Examples images are shown in Fig. 1 and Fig. 2.

1. Load the $T_{1}$-weighted sequence, adapt the slice orientation to provide a coronal or axial view with respect to the kidney (in scanner coordinates this is double-oblique) by importing the same geometry as for the anatomical (and $\mathrm{T}_{1}$ mapping sequence).

2. Set the required number of repetitions accordingly to the sampling time and to the duration of the dynamic acquisition. Typically, 50-100 repetitions provide enough points to be exploited by any of the analysis procedures.

3. Initiate the DCE-MRI scan.

4. After some baseline image acquisition (5-10 images before the injection are enough to derive average baseline values and signal oscillations) perform an intravenous bolus injection of the Gd-based CA through the tail vein at a dose of $0.02-0.1 \mathrm{mmol} \mathrm{Gd} / \mathrm{kg}$ b.w. by using a power injector to maintain the exact administration rate for each imaged mouse.

5. A demonstration of the signal intensity changes that can be expected for the DCE-MRI experiment across the kidneys is 


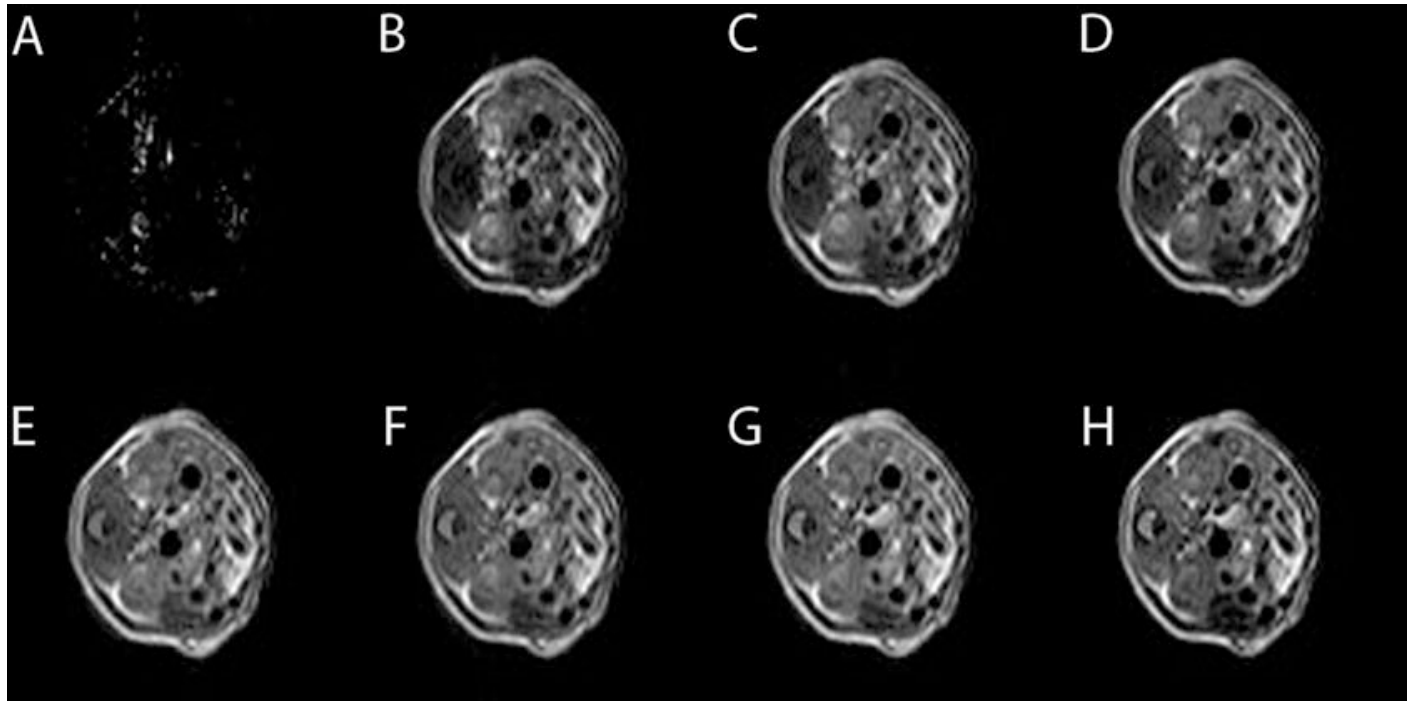

Fig. 1 Representative $T_{1}$-weighted images in kidneys obtained with the VTR scheme at different repetition times (TR) values: $75 \mathrm{~ms}$ (a), $534 \mathrm{~ms}$ (b), $1076 \mathrm{~ms}$ (c), $1738 \mathrm{~ms}$ (d), $2589 \mathrm{~ms}$ (e), $3782 \mathrm{~ms}$ (f), $5791 \mathrm{~ms}(\mathbf{g})$, and $15000 \mathrm{~ms}$ (h). Acquisition details are provided in Note $\mathbf{5}$

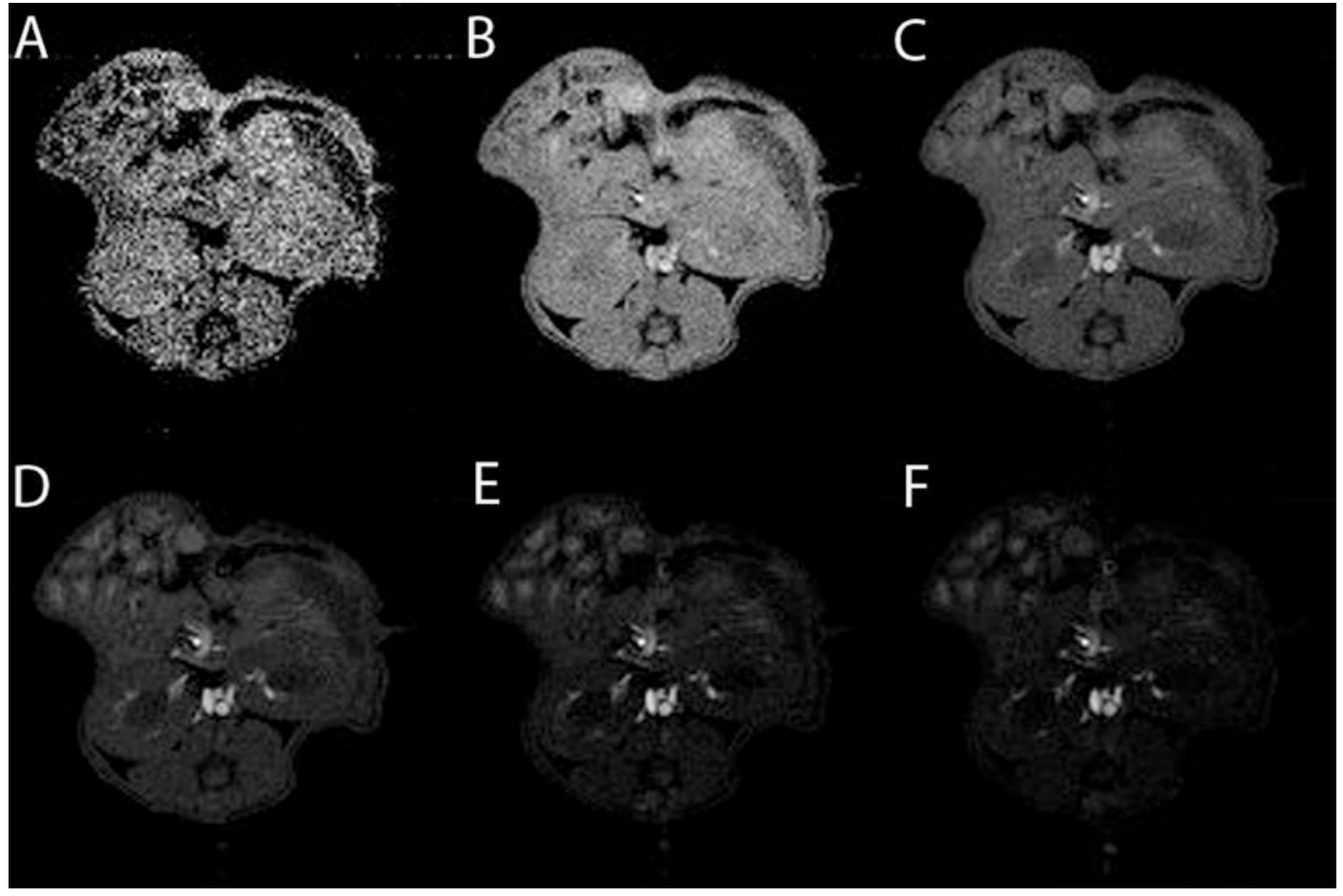

Fig. 2 Representative $T_{1}$-weighted images in kidneys obtained with VFA $T_{1}$ mapping approach by varying the excitation pulse flip angle values: $5^{\circ}$ (a), $15^{\circ}$ (b), $30^{\circ}$ (c), $45^{\circ}$ (d), $60^{\circ}$ (e), and $75^{\circ}$ (f) with a $7 \mathrm{~T} \mathrm{MRI} \mathrm{system}$. Acquisition details are provided in Note 6 


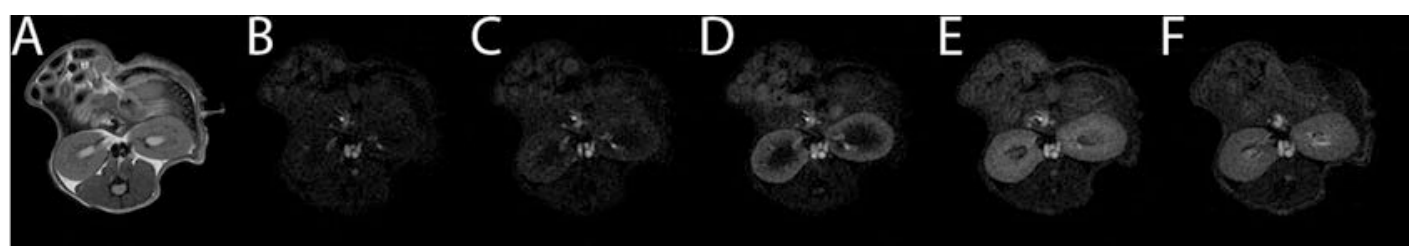

Fig. 3 Representative DCE-MRI images in kidneys (transversal orientation) at different time points following Gd-based contrast agent injection with a $7 \mathrm{~T}$ MRI scanner. Anatomical $\mathrm{T}_{2}$-weighted (a) and GRE-FLASH $\mathrm{T}_{1}$-weighted images before (b) and $4 \mathrm{~s}(\mathbf{c}), 30 \mathrm{~s}(\mathbf{d}), 5 \mathrm{~min}(\mathbf{e})$, and $10 \mathrm{~min}(\mathbf{f})$ after gadoteridol injection (dose $0.1 \mathrm{mmol} \mathrm{Gd} / \mathrm{kg}$ b.w.). Acquisition details are provided in Note 7

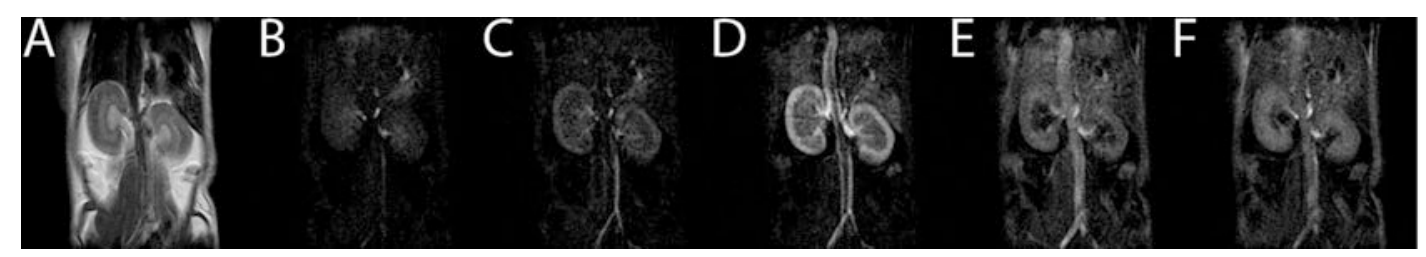

Fig. 4 Representative DCE-MRI images in kidneys (coronal orientation) at different time points following Gd-based contrast agent injection with a $7 \mathrm{~T}$ MRI scanner. Anatomical $\mathrm{T}_{2}$-weighted (a) and GRE-FLASH $\mathrm{T}_{1}$-weighted images before (b) and $4 \mathrm{~s}(\mathrm{bc}), 30 \mathrm{~s}(\mathbf{d}), 5 \mathrm{~min}(\mathbf{e})$, and $10 \mathrm{~min}(\mathbf{f})$ after gadoteridol injection (dose $0.1 \mathrm{mmol} \mathrm{Gd} / \mathrm{kg}$ b.w.). Acquisition details are provided in Note 8

given in Fig. 3 (axial orientation) and Fig. 4 (coronal orientation).

\section{Notes}

1. Intramuscular anesthesia can be administered as a mixture of xylazine $5 \mathrm{mg} / \mathrm{kg}$ (Rompun, Bayer, Italy) and tiletamine/zolepan $20 \mathrm{mg} / \mathrm{kg}$ (Zoletil 100, Virbac, Italy) with a dose dependent on the length of the MRI session. Usually, a 40-80 $\mu \mathrm{l}$ volume allows for a sleeping time of 30-60 min for a $25 \mathrm{~g}$ mouse.

2. A required dose for DCE-MRI in the kidneys is in the range $0.01-0.1 \mathrm{mmol} \mathrm{Gd} / \mathrm{kg}$ b.w. that would require too small volumes of the stock solution. Therefore, it is advisable to dilute stock solutions to ca. $50 \mathrm{mmol} / \mathrm{l}$. For instance, a $25 \mathrm{~g}$ mouse would receive $50 \mu \mathrm{l}$ of $50 \mathrm{mmol} / \mathrm{l} \mathrm{Gd}$-complex to achieve a dose of $0.1 \mathrm{mmol} \mathrm{Gd} / \mathrm{kg}$. Please avoid the inclusion of air bubbles in the catheter filled with the saline solution or with the contrast agent solution that will be injected into the animals.

3. DCE-MRI experiments are described for Bruker MRI systems, such as Avance 300 (7 T) and Biospec (3 T), (Bruker, Ettlingen, Germany) or for a Magnetom Skyra $3 \mathrm{~T}$ (Siemens Healthcare, Erlangen, Germany). Standard ${ }^{1} \mathrm{H}$ radiofrequency-coils 
(birdcage, quadrature) or phased-array ${ }^{\mathrm{l}} \mathrm{H}$ coils can be used for data reception.

4. Within the Bruker method, specific TR values can be suggested according to the estimated $\mathrm{T}_{1}$ values of the tissue.

5 . For estimated $\mathrm{T}_{1}$ of $1-3 \mathrm{~s}, 10 \mathrm{TR}$ values can be used $(580,810$, $1075,1380,1740,2180,2740,3525,4830$, and 10,000 ms) to correctly follow signal intensities recovery until plateau. With $10 \mathrm{TR}$ values complete acquisition lasted 8:40 min (for $96 \times 96$ matrix and 8 slices).

6 . For estimated $T_{1}$ values of $1-3 \mathrm{~s}$, we used six different $F A$ values $\left(5^{\circ}, 15^{\circ}, 30^{\circ}, 45^{\circ}, 60^{\circ}\right.$, and $\left.75^{\circ}\right)$. With a TR of $80 \mathrm{~ms}$ and matrix size of $96 \times 96$, the single image acquisition lasted more than $15 \mathrm{~s}$ ( 8 slices). The complete acquisition time of the VFA $\mathrm{T}_{1}$ map with six FA values is approximately $2 \mathrm{~min}$.

7. Example for DCE-MRI using a FLASH readout at $7 \mathrm{~T}$ : TR: $16 \mathrm{~ms}$; TE: $1.6 \mathrm{~ms}$; FA: $30^{\circ}$; scan averages: 2 ; slice thickness: $1.5 \mathrm{~mm}$; slice number: 1 ; slice orientation: axial; frequency encoding: head-feet; FOV $=30 \times 30 \mathrm{~mm}^{2}$; matrix size $=128 \times 128$; fat suppression $=$ on; acquisition time per image: $4 \mathrm{~s}$; number of repetitions: 150 .

8. Example for DCE-MRI using a FLASH readout at $7 \mathrm{~T}$ : TR: $15 \mathrm{~ms}$; TE: $1.7 \mathrm{~ms}$; FA: $30^{\circ}$; scan averages: 2 ; slice thickness: $1.5 \mathrm{~mm}$; slice number: 1 ; slice orientation: coronal; frequency encoding: head-feet; FOV $=30 \times 30 \mathrm{~mm}^{2}$; matrix size $=128 \times 128$; fat suppression $=$ on; acquisition time per image: $4 \mathrm{~s}$; number of repetitions: 150 .

9. Example for DCE-MRI using a 3D time-resolved angiography with stochastic trajectories (TWIST) sequence at $3 \mathrm{~T}$ : TR = $3.4 \mathrm{~ms}, \mathrm{TE}=1.4 \mathrm{~ms}, \mathrm{FA}: 20^{\circ}, \mathrm{FOV}=114 \times 50 \mathrm{~mm}^{2}$, generalized autocalibrating partially parallel acquisition (GRAPPA) of factor 2, matrix $=192 \times 84$; slice number: 2 , voxel resolution $=0.6 \times 0.6 \times 1.2 \mathrm{~mm}^{3}$. TWIST view sharing: $15 \%$ central region, $20 \%$ sampling density in the outer region, nominal temporal resolution $0.9 \mathrm{~s}$ per volume. Images were continuously acquired for $6 \mathrm{~min}$.

10. Mice can be alternatively anesthetized with intramuscular anesthesia, tail veins heated with hot water/heater equipment at $37^{\circ} \mathrm{C}$ and catheter placed in the animal.

11. It is important to monitor the respiration continuously throughout the entire experiment. 
The Italian Ministry for Education and Research (MIUR) is gratefully acknowledged for yearly FOE funding to the EuroBioImaging Multi-Modal Molecular Imaging Italian Node (MMMI).

This chapter is based upon work from COST Action PARENCHIMA, supported by European Cooperation in Science and Technology (COST). COST (www.cost.eu) is a funding agency for research and innovation networks. COST Actions help connect research initiatives across Europe and enable scientists to enrich their ideas by sharing them with their peers. This boosts their research, career, and innovation.

PARENCHIMA (renalmri.org) is a community-driven Action in the COST program of the European Union, which unites more than 200 experts in renal MRI from 30 countries with the aim to improve the reproducibility and standardization of renal MRI biomarkers.

\section{References}

1. Sawhney S, Mitchell M, Marks A, Fluck N, Black C (2015) Long-term prognosis after acute kidney injury (AKI): what is the role of baseline kidney function and recovery? A systematic review. BMJ Open 5(1):e006497. https://doi.org/10.1136/bmjopen-2014006497

2. Joannidis M, Druml W, Forni LG, Groeneveld ABJ, Honore PM, Hoste E, Ostermann M, Oudemans-van Straaten HM, Schetz M (2017) Prevention of acute kidney injury and protection of renal function in the intensive care unit: update 2017: expert opinion of the Working Group on Prevention, AKI section, European Society of Intensive Care Medicine. Intensive Care Med 43(6):730-749. https:// doi.org/10.1007/s00134-017-4832-y

3. Kline TL, Edwards ME, Garg I, Irazabal MV, Korfiatis P, Harris PC, King BF, Torres VE, Venkatesh SK, Erickson BJ (2018) Quantitative MRI of kidneys in renal disease. Abdom Radiol (NY) 43(3):629-638. https://doi.org/ 10.1007/s00261-017-1236-y

4. Zhang JL, Morrell G, Rusinek H, Sigmund EE, Chandarana H, Lerman LO, Prasad PV, Niles D, Artz N, Fain S, Vivier PH, Cheung AK, Lee VS (2014) New magnetic resonance imaging methods in nephrology. Kidney Int 85 (4):768-778. https://doi.org/10.1038/ki. 2013.361

5. Zhang JL, Rusinek H, Chandarana H, Lee VS (2013) Functional MRI of the kidneys. J Magn
Reson Imaging 37(2):282-293. https://doi. org/10.1002/jmri.23717

6. Mannelli L, Maki JH, Osman SF, Chandarana H, Lomas DJ, Shuman WP, Linnau KF, Green DE, Laffi G, Moshiri M (2012) Noncontrast functional MRI of the kidneys. Curr Urol Rep 13(1):99-107. https://doi. org/10.1007/s11934-011-0229-6

7. Attenberger UI, Morelli JN, Schoenberg SO, Michaely HJ (2011) Assessment of the kidneys: magnetic resonance angiography, perfusion and diffusion. J Cardiovasc Magn Reson 13:70. https://doi.org/10.1186/1532429X-13-70

8. Grenier N, Pedersen M, Hauger O (2006) Contrast agents for functional and cellular MRI of the kidney. Eur J Radiol 60 (3):341-352. https://doi.org/10.1016/j. ejrad.2006.06.024

9. Ebrahimi B, Textor SC, Lerman LO (2014) Renal relevant radiology: renal functional magnetic resonance imaging. Clin $\mathrm{J}$ Am Soc Nephrol. https://doi.org/10.2215/CJN. 02900313

10. Zollner FG, Konstandin S, Lommen J, Budjan J, Schoenberg SO, Schad LR, Haneder S (2016) Quantitative sodium MRI of kidney. NMR Biomed 29(2):197-205. https://doi. org/10.1002/nbm.3274

11. Zimmer F, Klotz S, Hoeger S, Yard BA, Kramer BK, Schad LR, Zollner FG (2017) Quantitative arterial spin labelling perfusion 
measurements in rat models of renal transplantation and acute kidney injury at $3 \mathrm{~T}$. $\mathrm{Z}$ Med Phys 27(1):39-48. https://doi.org/10.1016/ j.zemedi.2016.02.004

12. Hueper K, Peperhove M, Rong S, Gerstenberg J, Mengel $M$, Meier $M$, Gutberlet M, Tewes S, Barrmeyer A, Chen R, Haller H, Wacker F, Hartung D, Gueler F (2014) Tl-mapping for assessment of ischemia-induced acute kidney injury and prediction of chronic kidney disease in mice. Eur Radiol 24(9):2252-2260. https://doi.org/10. 1007/s00330-014-3250-6

13. Zollner FG, Schock-Kusch D, Backer S, Neudecker S, Gretz N, Schad LR (2013) Simultaneous measurement of kidney function by dynamic contrast enhanced MRI and FITCsinistrin clearance in rats at 3 tesla: initial results. PLoS One 8(11):e79992. https://doi. org/10.1371/journal.pone.0079992

14. Hueper K, Rong S, Gutberlet M, Hartung D, Mengel M, Lu X, Haller H, Wacker F, Meier M, Gueler F (2013) T2 relaxation time and apparent diffusion coefficient for noninvasive assessment of renal pathology after acute kidney injury in mice: comparison with histopathology. Investig Radiol 48(12):834-842. https://doi.org/10.1097/RLI. 0b013e31829d0414

15. Inoue $\mathrm{T}$, Kozawa $\mathrm{E}$, Okada $\mathrm{H}$, Inukai $\mathrm{K}$, Watanabe S, Kikuta $\mathrm{T}$, Watanabe $\mathrm{Y}$, Takenaka T, Katayama S, Tanaka J, Suzuki H (2011) Noninvasive evaluation of kidney hypoxia and fibrosis using magnetic resonance imaging. J Am Soc Nephrol 22 (8):1429-1434. https://doi.org/10.1681/ ASN.2010111143

16. Notohamiprodjo M, Reiser MF, Sourbron SP (2010) Diffusion and perfusion of the kidney. Eur J Radiol 76(3):337-347. https://doi.org/ 10.1016/j.ejrad.2010.05.033

17. Sourbron SP, Michaely HJ, Reiser MF, Schoenberg SO (2008) MRI-measurement of perfusion and glomerular filtration in the human kidney with a separable compartment model. Investig Radiol 43(1):40-48. https:// doi.org/10.1097/RLI.0b013e31815597c5

18. Prasad PV (2006) Evaluation of intra-renal oxygenation by BOLD MRI. Nephron Clin Pract 103(2):c58-c65. https://doi.org/10. $1159 / 000090610$

19. Dujardin M, Sourbron S, Luypaert R, Verbeelen D, Stadnik T (2005) Quantification of renal perfusion and function on a voxel-byvoxel basis: a feasibility study. Magn Reson Med 54(4):841-849. https://doi.org/10. $1002 / \mathrm{mrm} .20608$
20. Longo DL, Dastru W, Digilio G, Keupp J, Langereis S, Lanzardo S, Prestigio S, Steinbach O, Terreno E, Uggeri F, Aime S (2011) Iopamidol as a responsive MRI-chemical exchange saturation transfer contrast agent for $\mathrm{pH}$ mapping of kidneys: in vivo studies in mice at $7 \mathrm{~T}$. Magn Reson Med 65(1):202-211. https://doi.org/10. $1002 / \mathrm{mrm} .22608$

21. Longo DL, Busato A, Lanzardo S, Antico F, Aime S (2013) Imaging the $\mathrm{pH}$ evolution of an acute kidney injury model by means of iopamidol, a MRI-CEST pH-responsive contrast agent. Magn Reson Med 70(3):859-864. https://doi.org/10.1002/mrm.24513

22. Longo DL, Sun PZ, Consolino L, Michelotti FC, Uggeri F, Aime S (2014) A general MRI-CEST ratiometric approach for $\mathrm{pH}$ imaging: demonstration of in vivo $\mathrm{pH}$ mapping with iobitridol. J Am Chem Soc 136 (41):14333-14336. https://doi.org/10. $1021 /$ ja5059313

23. Longo DL, Cutrin JC, Michelotti F, Irrera P, Aime S (2017) Noninvasive evaluation of renal $\mathrm{pH}$ homeostasis after ischemia reperfusion injury by CEST-MRI. NMR Biomed 30(7). https://doi.org/10.1002/nbm.3720

24. Longo D, Aime S (2017) Iodinated contrast media as $\mathrm{pH}$-responsive CEST agents. In: Mcmahon MT, Gilad AA, JBM B, PCM VZ (eds) Chemical exchange saturation transfer imaging. Vol. Advances and applications. Pan Stanford Publishing, Singapore, pp 447-466. https://doi.org/10.1201/ 9781315364421-20

25. Wu Y, Zhou IY, Igarashi T, Longo DL, Aime S, Sun PZ (2018) A generalized ratiometric chemical exchange saturation transfer (CEST) MRI approach for mapping renal $\mathrm{pH}$ using iopamidol. Magn Reson Med 79 (3):1553-1558. https://doi.org/10.1002/ mrm.26817

26. Mariager CO, Nielsen PM, Qi HY, Schroeder M, Bertelsen LB, Laustsen C (2017) Can hyperpolarized C-13-urea be used to assess glomerular filtration rate? A retrospective study. Tomography 3(3):146-152. https://doi.org/10.18383/j.tom.2017. 00010

27. Duwel S, Hundshammer C, Gersch M, Feuerecker B, Steiger K, Buck A, Walch A, Haase A, Glaser SJ, Schwaiger M, Schilling F (2017) Imaging of $\mathrm{pH}$ in vivo using hyperpolarized (13)C-labelled zymonic acid. Nat Commun 8:15126. https://doi.org/10.1038/ ncomms 15126

28. Aime S, Botta M, Terreno E (2005) Gd(III)based contrast agents for MRI. Adv Inorgan 
Chem 57(57):173-237. https://doi.org/10. 1016/S0898-8838(05)57004-1

29. Sherry AD, Caravan P, Lenkinski RE (2009) Primer on gadolinium chemistry. J Magn Reson Imaging 30(6):1240-1248. https:// doi.org/10.1002/jmri.21966

30. Longo DL, Arena F, Consolino L, Minazzi P, Geninatti-Crich S, Giovenzana GB, Aime S (2016) Gd-AAZTA-MADEC, an improved blood pool agent for DCE-MRI studies on mice on $1 \mathrm{~T}$ scanners. Biomaterials 75:47-57. https://doi.org/10.1016/j.biomaterials. 2015.10.012

31. Geninatti-Crich S, Szabo I, Alberti D, Longo D, Aime S (2011) MRI of cells and mice at 1 and 7 Tesla with Gd-targeting agents: when the low field is better! Contrast Media Mol Imaging 6(6):421-425. https://doi.org/ $10.1002 / \mathrm{cmmi} .436$

32. Avedano S, Botta M, Haigh JS, Longo DL, Woods M (2013) Coupling fast water exchange to slow molecular tumbling in $\mathrm{Gd} 3+$ chelates: why faster is not always better. Inorg Chem 52 (15):8436-8450. https://doi.org/10.1021/ ic 400308 a

33. Botta M, Avedano S, Giovenzana GB, Lombardi A, Longo D, Cassino C, Tei L, Aime S (2011) Relaxometric study of a series of monoaqua Gd-III complexes of rigidified EGTA-like chelators and their noncovalent interaction with human serum albumin. Eur J Inorg Chem 6:802-810. https://doi.org/10. 1002 /ejic.201001103

34. Botta M, Tei L (2012) Relaxivity enhancement in macromolecular and nanosized GdIII-based MRI contrast agents. Eur J Inorg Chem 12:1945-1960. https://doi.org/10.1002/ ejic.201101305

35. Grenier N, Merville P, Combe C (2016) Radiologic imaging of the renal parenchyma structure and function. Nat Rev Nephrol 12 (6):348-359. https://doi.org/10.1038/ nrneph.2016.44

36. Winter KS, Helck AD, Ingrisch M, Staehler M, Stief C, Sommer WH, Braunagel $M$,
Kazmierczak PM, Reiser MF, Nikolaou K, Notohamiprodjo M (2014) Dynamic contrast-enhanced magnetic resonance imaging assessment of kidney function and renal masses: single slice versus whole organ/ tumor. Investig Radiol 49(11):720-727. https://doi.org/10.1097/RLI. 0000000000000075

37. Sourbron SP, Buckley DL (2013) Classic models for dynamic contrast-enhanced MRI. NMR Biomed 26(8):1004-1027. https://doi.org/ $10.1002 / \mathrm{nbm} .2940$

38. Zollner FG, Daab M, Sourbron SP, Schad LR, Schoenberg SO, Weisser G (2016) An open source software for analysis of dynamic contrast enhanced magnetic resonance images: UMMPerfusion revisited. BMC Med Imaging 16:7. https://doi.org/10.1186/s12880-016-01090

39. Beeman SC, Osei-Owusu P, Duan C, Engelbach J, Bretthorst GL, JJH A, Blumer KJ, Garbow JR (2015) Renal DCE-MRI model selection using Bayesian Probability Theory. Tomography $1(1): 61-68$. https:// doi.org/10.18383/j.tom.2015.00133

40. Zollner FG, Zimmer F, Klotz S, Hoeger S, Schad LR (2014) Renal perfusion in acute kidney injury with DCE-MRI: deconvolution analysis versus two-compartment filtration model. Magn Reson Imaging 32(6):781-785. https://doi.org/10.1016/j.mri.2014.02.014

41. Zöllner FG, Zimmer F, Klotz S, Hoeger S, Schad LR (2015) Functional imaging of acute kidney injury at 3 Tesla: investigating multiple parameters using DCE-MRI and a two-compartment filtration model. Z Med Phys 25(1):58-65. https://doi.org/10.1016/ j.zemedi.2014.01.002

42. Zollner FG, Weisser G, Reich M, Kaiser S, Schoenberg SO, Sourbron SP, Schad LR (2013) UMMPerfusion: an open source software tool towards quantitative MRI perfusion analysis in clinical routine. J Digit Imaging 26 (2):344-352. https://doi.org/10.1007/ s10278-012-9510-6

Open Access This chapter is licensed under the terms of the Creative Commons Attribution 4.0 International License (http://creativecommons.org/licenses/by/4.0/), which permits use, sharing, adaptation, distribution and reproduction in any medium or format, as long as you give appropriate credit to the original author(s) and the source, provide a link to the Creative Commons license and indicate if changes were made.

The images or other third party material in this chapter are included in the chapter's Creative Commons license, unless indicated otherwise in a credit line to the material. If material is not included in the chapter's Creative Commons license and your intended use is not permitted by statutory regulation or exceeds the permitted use, you will need to obtain permission directly from the copyright holder. 